\title{
Una respuesta institucional colombiana a niños/as que viven con VIH/SIDA
}

\author{
A Colombian institutional response \\ to Colombian children infected with HIV/AIDS
}

${ }^{1}$ Fundación Hospital de la Misericordia,

Departamento de Pediatría Universidad Nacional de Colombia. Carrera $7^{\mathrm{a}} \mathrm{N}^{\circ}$ 66-10/204. Bogotá D.C. Colombia. mibarrios@yahoo.com 2 Psicologia do Desenvolvimento, Instituto de Psicologia, Universidade Federal do Rio Grande do Sul.

\begin{abstract}
Children withHIV/AIDS is a social issue, the approach to which transcends the health sector. The normative, political, institutional and service sector responses have specificities related to children. A proposed approach is based on the following points: health care, nutrition, education, family-socioeconomic status, psychosocial repercussions, protection, housing, stigmatization and discrimination. Based on these guidelines, a survey was conducted of an institutional responseapplying the Bioecological Model of Human Development. Life of children in the institution is described and analyzed. A group of 31 children and 30 adults participated in this ethnographic study. The institutional response concerning health, nutrition, education and basic care, is satisfactory. The main weaknesses identified were nondisclosure of the diagnosis, stigmatization and discrimination, institutionalization validation, psychoemotional repercussions and stigmatization management, and the research budget. The children acknowledge that the institution satisfies their basic-needs and disapprove of some stigmatizing and coercive practices. The reintegration of the children with their families is recommended as a future strategy based on evidence that shows it has better bio-psychosocial results and lower costs.
\end{abstract}

Key words HIV/AIDS, Childhood, Institutional response, Social pediatrics
Resumen El VIH/SIDA en la niñez es un problema social y su abordaje trasciende al sector de la salud. Las respuestas normativas, políticas, institucionales $y$ de servicios tienen particularidades para la infancia. Un acercamiento propuesto se basa en los siguientes ejes: salud, nutrición, educación, condiciones socioeconómicas familiares, repercusiones psicosociales, protección, vivienda y estigmatización y discriminación. Desde estos lineamientos se exploró una institución usando la Teoría Bioecológica del Desarrollo Humano. Se describe y analiza la vida de niños/as en la institución. Es un estudio etnográfico y participaron 31 niños/as y 30 adultos/as. La respuesta institucional es satisfactoria en salud, alimentación, educación y cuidados básicos. La no revelación del diagnóstico, la estigmatización y discriminación contra los niños/as, la validación de la institucionalización, el manejo de las repercusiones psicoemocionales y del estigma y los costos del programa fueron las principales falencias. Los niños/as aprecian la respuesta institucional sobre el cubrimiento de sus necesidades básicas y rechazan algunas prácticas que ellos/as identifican como coercitivas y estigmatizantes. El reintegro de los niños/as a sus familias se recomienda como una estrategia de trabajo futuro sustentada en evidencias que demuestran mejores resultados biopsicosociales y bajo costo.

Palabras claves VIH/SIDA, Niñez, Respuesta Institucional, Pediatría Social 


\section{Introducción}

El VIH/SIDA se hace visible en la niñez por sus huérfanos. En 2005 se reportaron 995.000 huérfanos del SIDA en América Latina ${ }^{1}$. Para los niños/as huérfanas, infectadas o afectadas por el VIH/SIDA la respuesta institucional surge como una necesidad. Hay una relación del VIH/SIDA con la pobreza y la exclusión, así además de ser una enfermedad infecciosa es un problema social.

El VIH/SIDA comprende tres categorías en la niñez: infectados, afectados y vulnerables. Los infectados son quienes viven con el VIH. Los afectados son quienes no teniendo la infección sufren las consecuencias de la enfermedad. Así, son afectados los niños/as huérfanos del SIDA, quienes conviven con padres u otros familiares infectados por el VIH, etc. Son vulnerables quienes tienen alto riesgo de infectarse, como las víctimas de la violencia sexual.

La progresión de la infección en la niñez es más rápida que en los adultos y la enfermedad se aborda como un bloque: VIH/SIDA. Los antirretrovirales (TAR) se usan tempranamente.

Antes de la TAR efectiva en 1996 el curso de la infección del VIH en los niños/as era mortal ${ }^{2}$. El tratamiento cambió el significado de la enfermedad en la niñez y planteó nuevos retos, tales como abordar la infección como una enfermedad crónica o los desafíos que plantea la vida sexual durante la adolescencia y la adultez, entre otras. Así, el VIH/SIDA puede actualmente entenderse como una condición de vida y plantea nuevos retos para la sociedad, las políticas, los programas de salud y las instituciones vinculadas al cuidado de los niños que viven y conviven con el VIH/SIDA.

Los niños/as huérfanos y/o abandonados por causa del VIH/SIDA en Colombia son protegidos por el Instituto Colombiano de Bienestar Familiar (ICBF) o en instituciones especializadas en el tema. Históricamente son las ONG quienes han dado respuesta a la problemática infantil del VIH en Colombia.

\section{VIH/SIDA en la niñez: aprendizajes previos}

Para Brasil el Banco Mundial había previsto que en el año 2001, 1.200.000 personas estarían infectadas, sin embargo, en ese año solo se registraron 600.000 personas viviendo con el VIH/ SIDA $^{2}$.

Las bases del éxito del programa brasileño según Abadía, contemplan: una fuerte alianza entre el gobierno y la sociedad civil, la participación de "un Estado activista", el posicionamiento de los
Derechos Humanos y el Estado como garante de la salud como un derecho, asegurando el acceso universal y sin costo a las personas afectadas. Lo anterior fue posible por las negociaciones y los límites que impusiera el Estado a las compañías multinacionales que comercializan la TAR.

Hoy día se considera que las intervenciones efectivas y adecuadas para abordar el VIH/SIDA se deben fundamentar en dos principios esenciales: los Derechos Humanos, específicamente los Derechos Sexuales y/o Reproductivos y la disminución del estigma y la discriminación que sufren las personas que viven y conviven con el VIH/SIDA.

El tratamiento es el primer paso para disminuir el estigma y la discriminación. El estigma no sólo es un proceso de carácter individual sino que tiene significados sociales, de contexto e históricos que producen y reproducen las inequidades sociales y facilitan el sometimiento de las personas diferentes ${ }^{3}$. Así, el estigma se vivencia en la práctica en acciones concretas, que impiden a las personas estigmatizadas acceder a cosas especificas a las cuales tendrían derecho en su condición de seres humanos. Por ejemplo, a una atención digna y de calidad en una visita al servicio de salud.

La estigmatización y discriminación por el VIH/SIDA se potencializan con otros procesos estigmatizantes y discriminatorios asociados con clase social, etnia, raza, edad, género, orfandad, consumo de drogas, etc. Así, todos esos también participan en el rechazo.

Las construcciones sociales que determinan el estigma y la discriminación relacionados con el VIH/SIDA se imponen a la sociedad y sus individuos a través de discursos colectivos que impregnan a las personas a través de dispositivos institucionales. Así, se generan fenómenos de identificación colectiva e individual que hacen proclives a las personas de aceptar la exclusión social.

El VIH/SIDA tiene connotaciones sociales que trascienden a las relacionadas con el proceso salud-enfermedad-atención. La infección por el VIH en la práctica equivale a la enfermedad y lo anterior se determina social y culturalmente.

El camino de las instituciones brasileñas que trabajan con niños/as que viven y conviven con VIH/SIDA se dividen en tres momentos: a) La institucionalización para combatir la exclusión social; b) Las repercusiones familiares y sociales de la institucionalización; y c) La desinstitucionalización para garantizar la inclusión social ${ }^{3}$.

El cambio del accionar de las instituciones brasileñas se dio por la incorporación de los Derechos Humanos que privilegia el bienestar emo- 
cional garantizando la estancia de los niños con sus familias y evitando la estigmatización, discriminación y exclusión favorecida por la institucionalización. Así, se cambió la institucionalización por un soporte familiar y social.

En Colombia se ha avanzado menos, por eso las instituciones especializadas aún le apuestan a la institucionalización para trabajar el tema.

\section{VIH/SIDA en la niñez: apuestas actuales}

La respuesta al VIH/SIDA en la niñez debe tener un carácter amplio y garantizar, promover y restituir los Derechos del Niño. Incluye el compromiso de los gobiernos locales, la participación de la sociedad civil y de las personas y familias que viven y conviven con el VIH/SIDA.

Para regiones donde la infección es de baja prevalencia y concentrada, como en Colombia, UNICEF ha recomendado orientar la respuesta intersectorial e interdisciplinaria al VIH/SIDA en la niñez en lo siguiente: a) Salud, b) Nutrición, c) Condiciones socioeconómicas, d) Educación, e) Repercusiones psicosociales, f) Protección integral, g) Vivienda, y, h) Estigmatización ${ }^{1}$.

Estas recomendaciones pueden usarse para cualificar la oferta institucional y a partir de su análisis se pueden proponer cambios que permitan mejorar las condiciones y la calidad de vida de los niños/as que viven con VIH/SIDA.

Esta investigación se hizo realizando un trabajo etnográfico dentro de una institución especializada y tuvo el objetivo de describir y analizar la experiencia de vida de niños/as que viven y conviven con VIH/SIDA y su relación con la oferta institucional. Para ello se cualificó su situación de salud, nutrición, educación, condiciones socioeconómicas y de vivienda de sus familias, repercusiones psicoemocionales incluyendo el estigma y la discriminación, así como la respuesta institucional para cada uno de esos tópicos. Finalmente se hizo un balance entre las necesidades expresadas por los niños/as y la respuesta institucional.

\section{Método}

Esta es una investigación etnográfica cuyos datos fueron recogidos por dos de los investigadores dentro de la institución. La información recogida permitió comprender la complejidad de las relaciones sociales al interior de la institución ${ }^{4}$. La investigación cumplió con los requisitos legales de la institución y de la Universidad Nacional de Colombia, así como las normas legales y éticas.
La institución se localiza cerca a Bogotá. La población estudiada incluyó a todos los niños, niñas y preadolescentes que viven dentro de la institución, a las personas que trabajan en la Fundación, así como a quienes tenían relaciones directas con los niños. La población adulta participó mediante entrevistas o grupos focales con una muestra representativa de los distintos roles y según su disponibilidad para participar en el estudio.

Durante la investigación en la institución vivieron 15 niños y 16 niñas con edades entre los dos y los 13 años. La edad promedio fue de ocho años y la moda diez años. La institución operaba con 12 adultos en diferentes horarios, 11 mujeres y un hombre, con un tiempo promedio de trabajo de ocho horas.

Para el análisis el grupo de niños/as participantes fue subdivido en cuatro subgrupos: niños/as "pequeños" que tenían menos de cinco años, los/as "medianas" que tenían de seis a ocho años, niños/as “mayores”, quienes eran menores de diez años y preadolescentes a partir de esa edad.

La investigación incluyó cuatro fases. La planificación que duró tres meses. El trabajo de campo de seis meses, asistiendo entre tres a cuatro veces por semana, con un promedio de seis horas cada día. En un mes adicional se hicieron dos grupos focales. La fase de análisis e interpretación de los datos y la información incluyó un período de seis meses. La redacción y edición de este documento se hizo en diez meses. La pesquisa hizo parte del programa de estudios del Doctorado en Salud Pública del primer investigador y de Posgrado en Pediatría del segundo investigador. Ambos recibieron tutoría y asesoría de la tercera investigadora del artículo.

Se realizó revisión y análisis de los documentos de la institución que definen su filosofía y carácter llenándose una ficha específica. Se revisaron las carpetas institucionales de cada niño incluida su historia clínica.

Se hicieron 14 entrevistas semiestructuradas a niños/as, e igual número a los adultos. De los adultos entrevistados, diez pertenecían a la institución, dos al sector educativo, y dos fueron familiares. Se realizó un grupo focal con preadolescentes y el segundo con los adultos. Toda la información de las entrevistas y los grupos focales se transcribió y posteriormente se analizó en el programa ATLAS.ti versión 5.0 (Figura 1).

Aportes desde la Universidad Federal de Rio Grande del Sur en Porto Alegre, sirvieron para incorporar lineamientos de la Teoría Bioecológica del Desarrollo Humano de Urie Bronfenbrenner, la cual comprende el proceso del desarrollo 


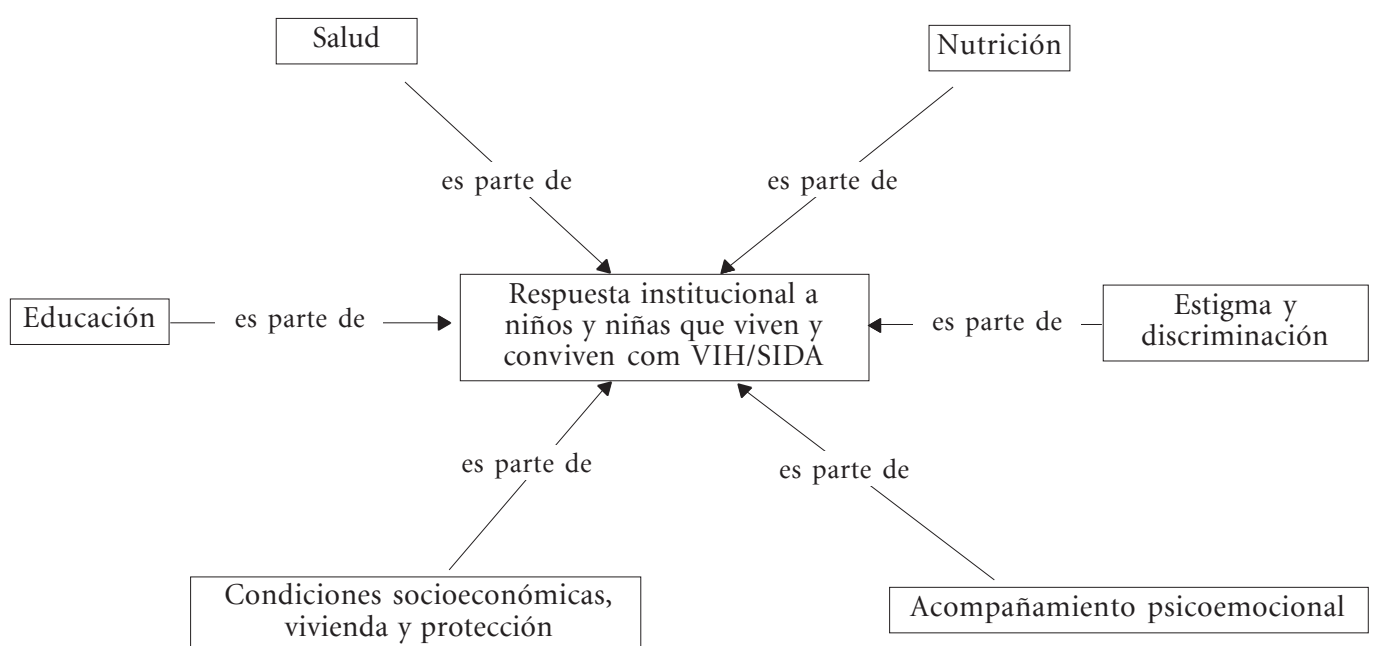

Figura 1. Componentes evaluados en la respuesta institucional para niños y niñas que viven y conviven con VIH/SIDA.

humano articulando cuatro dimensiones: persona, proceso, contexto y tiempo ${ }^{5}$.

La validez de los datos recogidos a través de la exploración etnográfica, así como en la aplicación metodológica de la inserción ecológica propuesta por Cecconello \& Koller ${ }^{6}$, se basa en gran medida en los vínculos que los investigadores generan con las personas participantes en virtud del compartir cotidiano, el respeto mutuo y la aceptación, entre otras, que dan garantía a la confiabilidad de los datos.

Siguiendo los anteriores lineamientos se construyó una matriz basada en el Modelo Bioecológico del Desarrollo Humano para estudiar el problema planteado: cualificar la experiencia de vida de niños/as que viven y conviven con VIH/SIDA en una institución cerrada y su relación con la oferta institucional.

El entendimiento de la vida institucional y en particular de la vivencia de los niños/as se hizo revisando la interacción que ellos/as tuvieron con las personas, los símbolos, los objetos, las rutinas y las actividades cotidianas. El componente personal tuvo en cuenta las interpretaciones, experiencias y realidades que cada niño/a hizo sobre las situaciones exploradas, así como su edad, sexo, salud, nutrición o enfermedad. El contexto se analizó tomando a la institución como un microsistema incluyendo las normas y dinámicas institucionales, la apropiación que los fun- cionarios y los niños hacen de los discursos y otros condicionantes generados desde otros contextos o las dinámicas de socialización entre los niños/as.

Como mesosistema se destaca en particular la relación con la escuela y como exo o macrosistema se incluyen entre otros, los discursos predominantes sobre el VIH/SIDA en la niñez, las políticas públicas sobre el tema, el enfoque biomédico de la enfermedad, el estigma y discriminación y las condiciones socioeconómicas de donde vienen los niños y las familias. El tiempo se contempló según el desarrollo de cada niño/a y comparando los cuatro grupos de edades previamente identificados.

Pragmáticamente, el análisis y la interpretación tuvieron en cuenta los lineamientos y normas institucionales, las experiencias de vida de los niños/as, las vivencias de los funcionarios (directivas, acompañantes y personal de apoyo), los espacios de conversación y reflexión, y las reflexiones de los investigadores.

\section{Resultados y Discusión}

Los hallazgos y reflexiones son complejas y reflejan el reto personal y social del vivir y convivir con el VIH/SIDA. 


\section{Caracterización institucional}

La institución es privada y se sostiene con aportes de particulares y empresas. Tiene una experiencia mayor de 10 años. Su origen y funcionamiento son de tipo religioso con preceptos caritativos: "nació como un hogar de paso para que los niños con VIH abandonados por sus familias o huérfanos pudieran tener un final digno, rodeados de amor, cuidado y aceptación. Sin embargo, gracias a la aparición de medicamentos antirretrovirales y al cuidado que se les prodiga a éstos niños, se debió reorientar su misión” [Documento de trabajo institucional].

"Es una organización que brinda a los niños/ as y jóvenes de escasos recursos y que viven con VIH/SIDA, un ambiente de amor y una atención integral... Así, en un futuro, podrán incorporarse a la sociedad de forma digna y productiva”. Este lineamiento misional se orienta a personas pobres (caridad) que tienen una condición de vida indigna (VIH/SIDA) para incorporarlos funcionalmente a la vida social (tiene implícito para los niños el no ser, el estar fuera de...) mediante un proceso productivo.

El programa es asistencial según sus objetivos: “a) Ofrecer un programa de atención integral de alta calidad, para atender las necesidades físicas, psicoafectivas y espirituales de los infantes que viven con el VIH/SIDA. b) Fomentar el programa de atención a sus padres y a su entorno social, con el objeto de informar y educar sobre el manejo de la enfermedad. c) Crear actitudes de solidaridad, compromiso y comprensión hacia las personas que padecen este flagelo".

Esta mezcla de "asistencialismo caritativo" limita el desarrollo integral de los niños/as por las exigencias que se les imponen. Un niño de 12 años expresa al respecto: “-¿Qué opinas del trato o del cuidado que te dan acá? -No me gusta, porque cada rato están pendientes de nosotros, no se pueden decir secretos. No me gusta que me manden a muchas cosas que yo no quiero hacer... toca pedir permiso para muchas cosas, para salir, para montar bicicleta. Tampoco me gusta que siempre para cada comida, toca hacer una fila, como si fuéramos bebés".

Para ingresar a la institución se requiere ser menor de 18 años, vivir con VIH, estar en situación de abandono y/o negligencia, o que la familia no tenga recursos económicos para sostenerlos. El funcionamiento institucional se rige autónomamente, el control por el ICBF es mínimo.

La participación del Estado en el cuidado de estos niños/as se efectúa principalmente a través de los servicios de salud y por el sector educativo. La escasa participación estatal limita el trato a los niños/as como sujetos titulares de derechos.

\section{Salud}

Veinte y dos de los niños y niñas de la institución (71\%) ingresaron antes de cumplir los cinco años al diagnosticarse VIH o SIDA por una infección severa asociada. El 90\% de los niños/as se infectaron por vía vertical. Se infiere de la revisión documental que menos de un $10 \%$ de las madres conocían que estaban infectadas. Lo cual es un indicador del mal control prenatal y que se correlaciona con la exclusión social de estas mujeres.

Lo anterior contrasta con reportes oficiales según los cuales de 2003 a 2007 se diagnosticaron 1127 gestantes con VIH, el 95,5\% de ellas recibieron profilaxis y $3,5 \%$ de ellas tuvieron hijos/as VIH positivos ${ }^{7}$.

Según ONUSIDA, Colombia es el tercer país de la región con menor porcentaje de pruebas de VIH para mujeres gestantes y tiene el segundo peor porcentaje de TAR para gestantes infectadas ${ }^{8}$.

En tres de los niños/as la infección se adquirió por otra vía. Uno por abuso sexual, otro por transfusión sanguínea y el otro por causa no determinada. Uno de los casos de transmisión vertical fue a través de la lactancia de una tía.

El VIH/SIDA es entendido por la institución como un problema de salud con implicaciones sociales, así la concepción biomédica predomina. De hecho, la mayoría del equipo que cuida a los niños/as son enfermeras.

Un niño de 11 años relata la asociación de la institución con un hospital: “-En qué te gustaría que cambiara la Fundación? -El color de la casa que parece un fantasma. Mira todo blanco, las paredes son blancas, las matas son blancas". Muchas de las dinámicas de la institución están condicionadas por los cuidados de la salud. El diario de campo consigna: "Para recibir los medicamentos, los niños se forman en fila en el pasillo. La enfermería es el sitio donde se preparan los medicamentos y les son administrados a los niños, aunque en ocasiones en un carro se van distribuyendo los medicamentos en cada habitación".

Durante la investigación todos los niños se hallaban recibiendo TAR. De los 31 niños/as, 14 (45\%) recibían tratamiento o profilaxis para otras infecciones como toxoplasmosis, pneumocistosis y/o tuberculosis. Todos los niños tenían acceso a los medicamentos, a controles clínicos y de laboratorio por especialistas en VIH/SIDA, como infectólogos pediatras, nutricionistas, etc. 
Esta atención se obtenía interdisciplinaria y regularmente.

La institución facilitó el acceso al tratamiento en las instituciones de salud. Se reportaron dificultades para la TAR y la realización de paraclínicos antes de la vinculación de los niños a la institución: "Yo estoy muy agradecida con la fundación porque desde que ella entró ha estado muy bien. A mí me demoraban mucho la droga y no me daban los más importantes". La institución reportó haber usado mecanismos jurídicos como la tutela para acceder a los medicamentos y laboratorios requeridos.

Estos datos evidencian las dificultades prácticas que viven los niños/as, las familias, e incluso las instituciones de protección para acceder al tratamiento integral de salud. Según el reporte oficial de diciembre de 2007, de 23.000 personas que requieren TAR en Colombia, solo $72 \%$ tienen cobertura (datos no diferenciados por edad) ${ }^{7}$.

Las condiciones de salud de los niños/as mejoraron desde el ingreso a la institución. El aspecto general de los niños es saludable y no parecen tener enfermedades crónicas o estigmatizantes. Sólo hay dos niñas de seis y diez años que tienen secuelas neurológicas secundarias a toxoplasmosis cerebral y meningitis bacteriana.

\section{Nutrición}

Un buen estado nutricional es importante para mantener la salud de estos niños/as. Al ingreso a la institución, 24 de los 31 niños/as presentaban algún grado de desnutrición (77\%). De los 31 seguidos/as, 14 (45\%) alcanzaron la fase SIDA y sólo uno de estos la desarrolló dentro de la institución. El 68\% de los niños desnutridos alcanzaron la fase de SIDA.

La desnutrición detectada al ingreso se atribuyó a las condiciones sociales de las cuales provenían estos niños/as, antes que a los efectos directos de la infección por el VIH. El 100\% de ellos venían de condiciones de pobreza con alta exclusión y vulnerabilidad social. Algunos padres tenían antecedentes de farmacodependencia, situación de calle, alcoholismo y estancia en prisión. Igualmente, algunas madres habían tenido infecciones de transmisión sexual durante la gestación, farmacodependencia, abuso sexual infantil y callejismo.

Los lactantes recibieron suplementación con leches de fórmula de acuerdo con la normatividad vigente en el país que garantiza el suministro de estas leches hasta los seis meses de edad para hijos/as de madres que viven con la infección ${ }^{9}$. Se infiere que más del 90\% de estos niños/as recibieron alimentación mixta durante sus primeros meses de vida antes del ingreso.

El 97\% de los niños/as presentó talla baja para su edad que es un indicador de desnutrición crónica, sin embargo, la mayoría presentó peso adecuado para la talla, lo cual sugiere un adecuado estado nutricional actual, en coherencia con la alimentación dentro de la institución. En general, se observó mejoría en los resultados de las evaluaciones nutricionales de los niños/as posteriores a su ingreso.

Se evidenciaron algunas percepciones y representaciones erradas sobre el tema de nutrición y el VIH. El relato de una familiar muestra este tipo de concepciones que también expresaron funcionarias: "a los niños que tienen VIH hay que cuidarlos, hay que darles la comida a sus horas, son comidas especiales, no se les puede dar calentado". La realidad es que este tipo de recomendaciones y preceptos carecen de bases científicas y son fundamentadas en las construcciones sociales de la enfermedad.

Otros lineamientos alimentarios que se aplican en la institución obedecen a otros determinantes sociales que trascienden los estándares y recomendaciones nutricionales. Por ejemplo, el diario de campo consigna: "Se les pide que guarden silencio durante las comidas, que coman todo lo que les sirven, independientemente de que les guste o no, y con la misma velocidad..." Estas regulaciones pueden tener relación con el paradigma caritativo asistencial que privilegia los preceptos institucionales.

\section{Educación}

Dos meses antes del trabajo de campo, la institución se mudó de un medio urbano a uno rural. Previo al desplazamiento, la mayoría de los niños/as mayores de cinco años estaban estudiando en colegios de la ciudad. La vinculación y adaptación de estos niños al nuevo colegio representó dificultades, retos y aprendizajes. Se generaron mecanismos de adaptación y resistencia a la estigmatización que se dio en la escuela, como lo ilustró una funcionaria:

"Ellos saben que no le pueden decir eso a todo el mundo [revelación del diagnóstico] y que no todo el mundo puede asimilar eso, porque mucha gente es ignorante al respecto y pueden señalarlos y rechazarlos, incluso hasta el personal médico. Ellos saben que pueden hablar del tema cuando hay confianza y se da pie para eso. Pero algunos han tenido problemas en el colegio y se 
ha llegado al punto en que ha tocado cambiarlos, porque incluso los molestan por el hecho de no tener papá ni mamá".

La vinculación al nuevo colegio planteó dificultades y revivió procesos de estigmatización. La institución intentó ingresar a los niños/as a la escuela rural pero no se logró. La directora de la escuela comentó: "intentar incluir a los niños en el colegio fue una complicación muy terrible... algunos papás (cuatro) retiraron a sus niños del colegio, y ya estaban incitando a otros ocho. Ellos decían que se les iba a prender el virus, que los iban a contagiar. Me dijeron: 'Entonces quédese con los niños enfermos"'.

Por lo anterior, se acondicionaron espacios institucionales como escuela y dos maestras del colegio iniciaron una intervención que se planteó como una estrategia de transición para la futura vinculación de los niños a programas formales de educación. Los menores de cinco años, así como las dos niñas con discapacidad carecieron de educación.

Las niñas/os mayores de ocho años se encontraban entre primero y quinto grado de educación básica primaria, es decir, tenían niveles importantes de "extra-edad escolar", lo cual es consistente con los reportes de la literatura. En este grupo de niños/as, el objetivo pedagógico fue su "nivelación" para que ingresaran a la escuela en los niveles que estaban cursando previamente. El trabajo con los niños/as "medianos" (cinco a ocho años) consistió en prepararlos para el ingreso al primer año de la educación básica primaria. De los 11 que iniciaron el proceso, en nueve de ellos se cumplieron los objetivos anteriores.

\section{Repercusiones psicoemocionales}

La literatura reporta que los niños/as que viven y conviven con el VIH/SIDA son susceptibles a presentar problemas psicológicos. Un niño de 12 años relata al respecto: “-Qué piensas de vivir con el VIH? -Es normal. Algunas veces es normal, otras veces no, por la medicina, y algunas veces me discriminan. -Quién te discrimina? -En el colegio. Por ejemplo, si una niña sabe, le cuenta al rector y el rector hace que empiecen a discriminarlo a uno.-Qué ocurre cuando te discriminan? -Por ejemplo, no juegan conmigo, o dicen: “¡ay!, mire a ese niño que tiene SIDA, no se junten con él porque se lo pega, ¡ay!, guácala”. -Te ha ocurrido eso? -No, ninguna vez".

El primero de estos elementos guarda relación con el proceso de identidad. Estos niños/as deben construir su mismidad sobre la base de sufrir una enfermedad que se asocia con contagiosidad. Este espejo que refleja el rechazo social, estigmatiza, discrimina y dificulta la construcción de un valor personal positivo. En el relato se lee cómo la elaboración psíquica del estigma no necesariamente está asociada con hechos reales.

Las dificultades que viven estos niños/as se incrementan por la no revelación del diagnóstico. Una funcionaria revela la política institucional al respecto: "La verdad con los pequeños aún no ha habido la oportunidad [de hablar sobre el VIH], yo pienso que no es como llegar a decir: "Bueno niños, vengan que les voy a dar una clase", sino que se suele hacer cuando van a ingresar al colegio, entonces se les cuenta cómo es la experiencia del colegio, que allí ya saben que son de la institución, entonces que si alguien les pregunta deben saber qué información dar, cuál no, cómo responder las preguntas, qué hacer si los llegan a rechazar o les llegan a hacer comentarios que les duelan, que les puedan lastimar, etc."

En 1999 la Academia Americana de Pediatría recomendó la revelación del diagnóstico a niños escolares y adolescentes que viven y conviven con VIH/SIDA ${ }^{10}$. Lo anterior ha reportado beneficios en el manejo de la enfermedad por los niños y adolescentes, familias e instituciones, así como disminución en la ansiedad de los padres, mejoría en los ajustes psicosociales de los niños/as, reducción de comportamientos problemáticos, incremento en la adherencia al tratamiento y mejor preparación para prácticas sexuales seguras. Por el contrario, el ocultamiento de la condición o una comunicación confusa y contradictoria se ha relacionado con estrés emocional, alteración de la imagen corporal y aislamiento social ${ }^{10}$.

Hoy se considera que la revelación del diagnóstico se debe hacer lo más pronto posible según el nivel de desarrollo y entendimiento del niño/a. En la institución, a pesar de que su trabajo es el apoyo a niños/as que viven con el VIH, no existe una política clara al respecto. El silencio es la regla y aunque varios de los niños/as saben de su condición, no se conversa sobre el tema.

Otra repercusión psicoemocional encontrada fue la sensación de vacío emocional por la ausencia o falta de contacto con la familia. Esto se evidencia especialmente en los niños/as mayores de cinco años, como reveló un niño de 12: “Cómo te impacta el hecho de que tu tengas ese virus? -Muy duro, porque les quita a su familia. Algunas veces el papá y la mamá mueren. A uno le da muy duro. -Tu papá o tu mamá han muerto por eso? -Los dos. Cuando yo era chiquito me dijeron: “Tu mamá se murió por VIH”. También 
mi papá murió por eso. - Te gusta vivir aquí? No, yo no quiero vivir aquí. Yo me quiero ir para mi casa. Porque me siento muy solo. -Por qué te sientes tan solo? -Por mi familia. Quisiera estar con mi familia."

La dinámica institucional, en particular las normas, rutinas y controles son fuentes de malestar emocional, como lo expresa una niña de 10 años: "Yo por qué toda una verraca vida en una fundación, yo no quiero volver a la fundación". Los datos encontrados no permiten establecer correlaciones entre los estadios de la enfermedad y las afectaciones emocionales encontradas. En cuanto al tratamiento de los problemas psicológicos éstos son abordados individualmente, $y$ en respuesta a crisis emocionales, en los servicios de salud.

\section{Condiciones socioeconómicas, vivienda y protección}

Se ratificó la relación que existe entre el VIH y la vulnerabilidad social, la cuál es más que pobreza. El VIH/SIDA en los progenitores de los niños deterioró la dinámica familiar y las condiciones económicas. Los gastos por la atención en salud fueron responsables de la institucionalización en algunos niños/as. El deterioro de los ingresos familiares por causa del VIH también ocasionó la vinculación de los hermanos de los niños/as al trabajo.

En 22 de los 31 niños/as (71\%) se conserva el vínculo familiar y el grupo familiar no recibe apoyo económico del Estado, lo cual condicionó la institucionalización. La mayoría de estos niños/ as se vinculó a la institución por la gestión de los hospitales donde se hizo su diagnóstico. Es decir que, las respuestas y resultados obtenidos fueron ajenos a las políticas públicas en el tema.

La respuesta de la institución se basa en la protección infantil originada en la Carta de Ginebra de 1924, la cual condicionó la acepción del "menor como objeto de protección" 2006 mediante el Código de Infancia y Adolescencia, en Colombia se adopta jurídicamente la doctrina de la protección integral basada en los Derechos del $\mathrm{Niño}^{12}{ }^{12}$ Sin embargo, existe camino entre la sanción de una ley y su aplicación en las prácticas institucionales y privadas.

La doctrina del "menor como objeto de protección" se basa en una perspectiva de necesidades, se piensa que el niño merece o necesita ayuda. En cambio, desde la perspectiva de derechos, se garantiza que el niño tiene el derecho a recibir ayuda. La institución estudiada facilita satisfactoriamente salud, la educación o la alimentación, sin embargo, otros procesos de su desarrollo integral, como la participación o niveles de autonomía acordes con su desarrollo, son usualmente negados. Al respecto, se destaca la ausencia de consulta o participación de cualquier tipo en la decisión tomada por la institución sobre el cambio de sede, hecho que afectó directamente la vida de ellos/as.

Según la institución el costo mensual aproximado por atención de cada niño es 800 dólares, que contrasta con el origen humilde de cada niño/ a. Una funcionaria comenta: "los niños reciben muchas cosas, tienen televisor, equipo de sonido, muchos regalos, y a veces regalos muy caros, pero no tienen personas que estén aquí para jugar con ellos. Necesitan personas que realmente se interesen en ellos, en quién son, en qué piensan, en qué sienten, no en que quieren tal cosa. Obviamente, si tú le preguntas a un niño, él quiere el carro, pero entonces hay que enseñarle que las cosas no son lo más importante, y también pienso que no es lo mejor para ellos, porque ellos son pobres. Ellos tienen la impresión que si rompen una cosa no pasa nada, pero cuando sean adultos, van a tener que trabajar para ganar plata, y no sé qué tipos de trabajo van a hacer. Estudiar en una universidad es muy caro, y después de aquí, no les van a pagar la universidad, no pueden. Entonces, ¿qué tipo de trabajos van a tener?, ¿qué tipo de vida?, y qué desilusión para ellos. Hay un contraste muy terrible en sus vidas".

Este contraste fue denominado por AbadíaBarrero como "enfermedades de la pobreza-respuestas privilegiadas"13. Durante la exploración la institución manifestó la intención de incrementar el número de beneficiarios porque su capacidad instalada es de 80 cupos.

Lo anterior es contrario a las recomendaciones para trabajar el VIH en la infancia en países de prevalencia baja y con epidemia concentrada ${ }^{1}$. La evidencia ha documentado que la forma más barata, efectiva y armónica de apoyar a estos niños/ as es mantenerlos en sus hogares o con la familia extensa, y que la institucionalización deba ser lo último. Las abuelas/os son el principal apoyo para estos niños/as cuando los progenitores desaparecen. El apoyo y el trabajo con estas personas $u$ otros familiares se deben constituir en una línea de intervención sobre este problema en el país.

\section{Estigma y discriminación}

La OMS ha declarado que "la discriminación es un peligro para la salud pública y la estigmatización es, en sí misma, una amenaza para el bien- 
estar social"14. El estigma y la discriminación se construyen desde las representaciones que tiene la sociedad sobre el VIH/SIDA.

Una funcionaria de la institución expresa: "los genes no se niegan, obviamente se sabe que el VIH puede darle a cualquier persona, pero la regla general aquí es que hay muchas señoras que han sido inestables con su pareja y lo mismo los señores. Desafortunadamente como se hereda lo bueno, también se hereda lo malo, y aquí hay niñas que ya se les nota muchas actitudes de sus madres, y se ve que van a ser re-coquetas y refregadas en el amor".

Así, la lucha contra la estigmatización y discriminación que sufren los niños/as y las familias que viven y conviven con el VIH es un reto a trabajar. Las raíces de estos fenómenos son usualmente inconscientes y naturalizadas, por tanto, trabajos reflexivos que promuevan la emancipación de los individuos y los grupos señalan un camino a seguir.

\section{Consideraciones finales}

El VIH/SIDA es un problema prioritario para las políticas de salud. Lo anterior se consolidó a partir del establecimiento de los Objetivos de Desarrollo del Milenio de la ONU. En Colombia, hay avances en aspectos normativos, técnicos y asistenciales sobre el VIH/SIDA. Así, las metas relacionadas con el VIH/SIDA son las únicas que se han alcanzado dentro de las contempladas en la Política Nacional de Salud Sexual y Reproductiva.

En contraste con lo anterior, el VIH/SIDA en la niñez en Colombia tiene menos avances. La exploración realizada en una institución privada especializada en el tema, permite inferir algunas conclusiones preocupantes. A pesar de los lineamientos normativos e institucionales, el tema del VIH/SIDA en la niñez no está posicionado en la agenda pública. La respuesta que se está dando al mismo es sectorizada y se brinda principalmente desde el sector de la salud. En el sistema de protección integral la respuesta es débil. El sector educativo carece de elementos y lineamientos para dar respuesta a las necesidades educativas de estos niños/as, en particular para trabajar y combatir el estigma y la discriminación.

En las familias exploradas, se manifestó su incapacidad para suplir las necesidades de sus niños/as conexas con el vivir y convivir con el VIH/SIDA. Por lo anterior, una respuesta institu- cional de carácter privado y con un enfoque de trabajo caritativo-asistencial se erigió en sus historias de vida como una alternativa social adecuada. Sin embargo, esto deja ver una falta de responsabilidad estatal sobre la situación del VIH/SIDA en la niñez.

La respuesta brindada a los niños/as abordados es satisfactoria con relación al cumplimiento de las necesidades en salud, alimentación y educación. Pero los paradigmas institucionales de trabajo coincidentes con el "menor como objeto de protección” y no con la protección integral basada en el niño como sujeto titular de derechos, limitan los resultados en algunas áreas. Se evidenciaron limitantes para fomentar el desarrollo integral, la autonomía y su construcción como sujetos sociales, que teniendo una condición de vida especial: el vivir con VIH/SIDA, tienen grandes retos que superar.

La falta de política institucional y la práctica del silencio sobre la revelación del diagnóstico, la reproducción de los fenómenos de estigmatización y discriminación contra los niños/as, la validación de la institucionalización, el poco acompañamiento y manejo de las repercusiones psicoemocionales y del estigma, y los costos del programa se evidenciaron como las principales deficiencias.

El balance evaluativo de la vida institucional por parte de los niños/as se mueve entre dos polos. Por un lado, hay reconocimiento y agradecimiento por el cubrimiento de sus necesidades básicas. Para quienes conocen el diagnóstico, se valora lo que se realiza sobre el cuidado de su salud. Y por el otro, se cuestionan y rechazan algunas dinámicas y normas de la institución que son entendidas por ellos y ellas como coercitivas (las que tienen que ver con el control) y estigmatizantes (aquellas asociadas con la percepción de la institución como un "medio hospitalario"). El trabajo de la institución poco cuestiona y aborda, por sus mismas limitaciones y misión, los determinantes y problemáticas sociales asociadas con el VIH/SIDA en la niñez. Por lo anterior, el reintegro de los niños/as a sus entornos sociales y familiares no se plantea como una meta futura, lo cual está en oposición con las recomendaciones vigentes del manejo del VIH en la niñez.

Finalmente, la exploración permite ratificar que la carta de navegación dada por los Derechos del Niño debe ser la guía central a seguir para el abordaje integral, multi e interdisciplinario y especialmente social y político del VIH en la niñez. 


\section{Colaboradores}

MEB Acosta, JGD Amaya y SH Koller participaron igualmente de todas las etapas de elaboración del artículo.

\section{Agradecimientos}

Agradecemos a los niños y las niñas de la institución por permitirnos acercarnos y compartir sus vidas, ellos son el motivo principal de esta investigación. A los directivos/as y funcionarios/as de la institución por reflexionar con nosotros sobre la dinámica institucional. A todas las personas en quienes nos hemos apoyado para la realización de este trabajo, principalmente a nuestras familias y amigos/as, con quienes dejamos de compartir espacios y momentos para concluir esta exploración.

\section{Referências}

1. Quality Assurance Project, USAID, Health Care Improvement Project, UNICEF. The Evidence Base for Programming for Children Affected by HIV/AIDS in Low Prevalence and Concentrated Epidemic Countries. Quality Assurance Project, USAID, Health Care Improvement Project, and UNICEF; 2008 [consultado en 2012 dic 2]. Documento electrónico disponible en: http://www.hciproject.org/sites/default/files/OVC \%20Evidence_Working\%20Paper_April\%202008.pdf

2. Abadía-Barrero C. Sida y niñez en Brasil: respuestas sociales que promueven la madurez de los derechos humanos. Venezuela: Universidad Central de Venezuela; 2006.

3. Abadía-Barrero C, Castro A. Experiences of stigma and access to HAART in children and adolescents living with HIV/AIDS in Brazil. Social Science and Medicine 2006; 62(5):1219-1228.

4. Guber R. La etnografía: Método, campo y reflexividad. Bogotá D.C.: Editorial Norma; 2006.

5. Bronfenbrenner U. On Making Human Beings Human. Bioecological Perspectives on Human Development. California: SAGE Publications; 2005.

6. Cecconello A, Koller S. Inserção Ecológica na Comunidade: Uma Proposta Metodológica para o Estudo de Famílias em Situação de Risco. Psicologia: Reflexão e Crítica 2003; 16(3):515-524.

7. Colombia. Ministerio de la Protección Social República. Resumen de la situación de la epidemia por VIH/SIDA en Colombia Diciembre 2007. Re pública de Colombia; 2007 [consultado 2009 nov]. Documento electrónico disponible en: http://www. onusida.org.co/RESUMEN_DATOS_SIDA_1_ dic.pdf.

8. ONUSIDA, Organização Mundial de Saúde (OMS. Situación de la epidemia de SIDA: Diciembre de 2006. ONUSIDA, OMS; 2006 [consultado en 2012 dic 2]. Disponible en: http://data.unaids.org/pub/ EpiReport/2006/2006_EpiUpdate_es.pdf.
9. Grupo de Antropología Médica Crítica-Universidad Nacional de Colombia, ICBF, Save the Children, UNICEF. Derechos de los niños, niñas y adolescentes afectados por el VIH SIDA en Colombia Estudio de casos en Cúcuta, Pereira y Villavicencio. Bogotá D.C.: UNICEF; 2009.

10. Abadía-Barrero C, Larusso M. The Disclosure Model versus a Developmental Illness Experience Model for Children and Adolescents Living with HIV/AIDS in São Paulo, Brazil. AIDS PATIENT CARE and STDs 2006; 20(1):36-43.

11. Galvis L. Las niñas, los niños y los adolescentes. Titulares activos de derechos. Bogotá D.C.: Ediciones Aurora; 2006.

12. República de Colombia. Ministerio de la Protección Social, e Instituto Colombiano de Bienestar Familiar. ABC Código de la Infancia y de la Adolescencia. Ley 1098 de 2006. Bogotá: Imprenta Nacional de Colombia; 2006.

13. Abadía-Barrero C. Growing up in a world with AIDS: social advantages of having AIDS in Brazil. AIDS CARE 2002; 14(3):417-423.

14. Luque R. El SIDA en primera persona: información y claves para el logro de acuerdos preventivos. Bogotá D.C.: Panamericana Editorial; 2001.

Artigo apresentado em 31/05/2011

Aprovado em 15/09/2012

Versão final aprovada em 11/10/2011 